\title{
The Solution of the Cosmological Constant Problem: The Cosmological Constant Exponential Decrease in the Super-Early Universe
}

\author{
$\mathrm{Ol}^{\prime}$ ga Babourova ${ }^{1}$ and Boris Frolov ${ }^{2, *}$ \\ 1 Department 'Physics', Moscow Automobile and Road Construction State Technical University (MADI), \\ Leningradsky pr., 64, 125319 Moscow, Russia; babourova@mail.ru \\ 2 Institute of Physics, Technology and Information Systems, Moscow Pedagogical State University (MPGU), \\ M. Pirogovskaya ul., 29, 119992 Moscow, Russia \\ * Correspondence: frolovbn@mail.ru or bn.frolov@mpgu.su; Tel.: +7-903-267-7107
}

Received: 30 September 2020; Accepted: 29 November 2020; Published: 4 December 2020

\begin{abstract}
The stage of a super-early (primordial) scale-invariant Universe is considered on the basis of the Poincaré-Weyl gauge theory of gravity in a Cartan-Weyl space-time. An approximate solution has been found that demonstrates an inflationary behavior of the scale factor and, at the same time, a sharp exponential decrease in the effective cosmological constant from a huge value at the beginning of the Big Bang to an extremely small (but not zero) value in the modern era, which solves the well-known "cosmological constant problem."
\end{abstract}

Keywords: Poincaré-Weyl gauge theory; super-early (primordial) Universe; effective cosmological constant

\section{Introduction}

In [1], the gravitational field as the field of curvature and torsion was introduced as the gauge field of the localized Poincaré group. This led to the construction of the Einstein-Cartan theory of gravity [2], based on the Lagrangian function in the form of a curvature scalar of the Riemann-Cartan space.

The next step towards the construction of a Poincaré-gauge theory of gravity was made in [3-5], where the Lagrangian quadratic in the curvature tensor was introduced, and the theorem on the sources of a gauge field was formulated and proven in a general form, as well as in $[6,7]$, where in addition, the Lagrangian quadratic in the torsion tensor was also introduced (for the Poincaré-gauge theory of gravity, see [8-12]).

Then, in [13-17], the Poincaré-gauge local symmetry was supplemented by transformations of the Weyl symmetry subgroup - stretching and contraction (dilatations) of space-time. In [15-17], the gauge theory of the Poincaré-Weyl group was developed. In this theory, tetrad coefficients (which cannot be gauge fields, since they are tensor components), as well as connection, are some functions of real translational, rotational, and dilatational gauge fields.

It has been shown that with the Poincaré-Weyl gauge theory of gravitation, the space-time acquires the properties of a Cartan-Weyl space with a curvature 2-form $\mathcal{R}^{a}{ }_{b}$ and a torsion 2-form $\mathcal{T}^{a}$, as well as a nonmetricity 1 -form $\boldsymbol{Q}_{a b}$ with the Weyl condition, $\boldsymbol{Q}_{a b}=(1 / 4) g_{a b} \boldsymbol{Q}$. In this case, in addition to the metric tensor, a scalar field $\beta$ appears, which by its properties coincides with the scalar field introduced earlier by Dirac [18].

In [19-22], it was hypothesized that the group of symmetry of space-time at the primordial stage of the evolution of the Universe- the epoch of the Big Bang and subsequent inflation-was not the Poincare group, but the Poincaré-Weyl group. At this stage, the rest masses of elementary particles had not yet appeared, and all interactions were carried out by massless quanta; therefore, these interactions had the property of scale invariance. 
The proposed hypothesis is based on the assumption made by Harrison and Zel'dovich about the approximate scale invariance of the early stage of the evolution of the Universe, which underlies the calculation of the initial part of the spectrum of primary fluctuations of matter density in the early Universe (Harrison-Zel'dovich spectrum, see [23]) and has been confirmed by results of the COBE experiment for the study of the anisotropy of the brightness of the background radiation (see [24]).

This paper presents preliminary results of a possible solution to the problem of the cosmological constant, which is one of the most important problems of modern fundamental physics. The solution of this problem will allow to reconcile the theory of the evolution of the Universe with modern physical concepts.

\section{Lagrangian Density}

If we omit the terms with squares of curvature, the Lagrangian density 4-form of the Poincaré-Weyl gauge theory of gravity takes the form [20-22]:

$$
\begin{aligned}
\mathcal{L} & =2 f_{0} \beta^{2}\left[(1 / 2) \mathcal{R}^{a}{ }_{b} \wedge \eta_{a}{ }^{b}-\beta^{2} \Lambda_{0} \eta+\rho_{1} \mathcal{T}^{a} \wedge * \mathcal{T}_{a}+\rho_{2}\left(\mathcal{T}^{a} \wedge \theta_{b}\right) \wedge *\left(\mathcal{T}^{b} \wedge \theta_{a}\right)\right. \\
& +\rho_{3}\left(\mathcal{T}^{a} \wedge \theta_{a}\right) \wedge *\left(\mathcal{T}^{b} \wedge \theta_{b}\right)+\xi Q \wedge * Q+\zeta Q \wedge \theta^{a} \wedge * \mathcal{T}_{a}+l_{1} \beta^{-2} \mathrm{~d} \beta \wedge * \mathrm{~d} \beta \\
& \left.+l_{2} \beta^{-1} \mathrm{~d} \beta \wedge \theta^{\alpha} \wedge * \mathcal{T}_{\alpha}+l_{3} \beta^{-1} \mathrm{~d} \beta \wedge * Q\right]+\beta^{4} \Lambda^{a b} \wedge\left(Q_{a b}-(1 / 4) g_{a b} Q\right),
\end{aligned}
$$

where $\theta^{a}(a=0,1,2,3)$ are basis 1 -forms, * is the Hodge dualization operation, $\Lambda^{a b}$ are the Lagrange multipliers, $\eta$ is a volume 4-form with components $\eta_{a b c d}$, and $\eta_{a b}=(1 / 2 !) \theta^{c} \wedge \theta^{d} \eta_{a b c d}$.

According to Gliner $[25,26]$, the cosmological constant $\Lambda$ in the Einstein equations is interpreted as the energy of the hypothetical vacuum-like medium, which is currently called dark energy. The term $\beta^{2} \Lambda_{0}$ in (1) describes the effective cosmological constant [20-22] (interpreted here as dark energy), depending on the Weyl-Dirac scalar field $\beta$ ( $\Lambda_{0}$ is the theory parameter providing the correct rate of inflation). The equality $\beta_{0}^{2} \Lambda_{0}=10^{120} \Lambda$ (see [27]) should be fulfilled, where $\Lambda$ is the modern value of the cosmological constant and $\beta_{0}$ is the value of the Weyl-Dirac scalar field at $t=0$.

\section{The Derivation of the Field Equations and Its Consequences}

The derivation of the variational field equations is based on the use of the following lemma on commutation of variation and dualization operations of external forms in the Cartan-Weyl space, proved in $[20,28]$ :

Lemma 1. Let $\Phi$ and $\Psi$ be arbitrary $p$-forms on n-dimensional manifold, then the following relation holds for the commutator of variation and the operation of dual Hodge conjugation:

$$
\begin{aligned}
\Phi \wedge \delta * \Psi & =\delta \Psi \wedge * \Phi \\
& +\delta g_{\rho}\left(\frac{1}{2} g^{\rho} \Phi \wedge * \Psi+(-1)^{p(n-1)+s+1} \theta^{\sigma} \wedge *\left(* \Psi \wedge \theta^{\rho}\right) \wedge * \Phi\right)+ \\
& +\delta \theta^{\sigma} \wedge\left((-1)^{p} \Phi \wedge *\left(\Psi \wedge \theta_{\sigma}\right)+(-1)^{p(n-1)+s+1} *\left(* \Psi \wedge \theta_{\sigma}\right) \wedge * \Phi\right) .
\end{aligned}
$$

here, $n=4$ is the dimension of space-time and $s=3$ is the index of the metric form of the Minkowski space.

Then, this Lemma was modified, taking into account the presence of the scalar Weyl-Dirac field, and the following important relationships were obtained $\left(\Phi^{a}{ }_{b}, \Phi_{a}, \Phi^{a b}\right.$ are arbitrary 2-forms) [20]:

$$
\begin{gathered}
\delta \mathcal{T}^{a} \wedge\left(f(\beta) \Phi_{a}\right)=\mathrm{d}\left(\delta \theta^{a} \wedge f(\beta) \Phi_{a}\right)+\delta \theta^{a} \wedge \mathrm{D}\left(f(\beta) \Phi_{a}\right)+\delta \Gamma^{a}{ }_{b} \wedge \theta^{b} \wedge f(\beta) \Phi_{a} \\
\delta Q_{a b} \wedge\left(f(\beta) \Phi^{a b}\right)=\mathrm{d}\left(-\delta g_{a b} f(\beta) \Phi^{a b}\right)+\delta g_{a b} \mathrm{D}\left(f(\beta) \Phi^{a b}\right)+\delta \Gamma^{a}{ }_{b} \wedge 2 f(\beta) \Phi_{(a}{ }^{b)} .
\end{gathered}
$$


We present here the result of using Lemma to vary some terms of the Lagrangian density (1):

$$
\begin{aligned}
& \delta\left(\frac{1}{2} \beta^{2} \mathcal{R}^{a}{ }_{b} \wedge \eta_{a}{ }^{b}\right)=\mathrm{d}\left(\delta \Gamma^{a}{ }_{b} \wedge \frac{1}{2} \beta^{2} \eta_{a}{ }^{b}\right)+\beta \mathrm{d} \beta \mathcal{R}^{a}{ }_{b} \wedge \eta_{a}{ }^{b}+ \\
+ & \delta \Gamma^{a}{ }_{b} \wedge \beta^{2}\left(-\frac{1}{4} \mathrm{Q} \wedge \eta_{a}{ }^{b}+\frac{1}{2} \mathrm{~T}_{c} \wedge \eta_{a}{ }^{b c}+\frac{1}{2} \eta_{a c} \wedge \mathrm{Q}^{b c}+\mathrm{d} \ln \beta \wedge \eta_{a}{ }^{b}\right)+ \\
+ & \delta g_{a b}\left(\frac{1}{4} g^{a b} \beta^{2} \mathcal{R}^{c}{ }_{d} \wedge \eta_{c}{ }^{d}+\frac{1}{2} \beta^{2} \theta^{(a} \wedge \theta_{c} \wedge * \mathcal{R}^{|c| b)}\right)+\delta \theta^{a}\left(\frac{1}{2} \beta^{2} \mathcal{R}^{b}{ }_{c} \wedge \eta_{b}{ }^{c} a\right), \\
\delta\left(\beta^{4} \Lambda_{0} \eta\right)= & 4 \delta \beta \beta^{3} \Lambda_{0} \eta+\delta \theta^{a} \wedge \beta^{4} \Lambda_{0} \eta_{a}, \quad \eta_{a}=\frac{1}{3} \theta^{b} \wedge \eta_{a b}=\frac{1}{3 !} \theta^{b} \wedge \theta^{c} \wedge \theta^{d} \eta_{a b c d} . \\
& \delta(\mathrm{d} \beta \wedge * \mathrm{~d} \beta)=-\delta \beta \cdot 2(\mathrm{~d} * \mathrm{~d} \ln \beta+\mathrm{d} \ln \beta \wedge \mathrm{d} \ln \beta)+\mathrm{d}(2 \delta \beta * \mathrm{~d} \beta)+ \\
& +\delta g_{a b}\left(\left(\frac{1}{2} g^{a b} \mathrm{~d} \beta-\theta^{(a} \wedge *\left(* \mathrm{~d} \beta \wedge \theta^{b)}\right)\right) \wedge * \mathrm{~d} \beta\right)+ \\
& +\delta \theta^{a} \wedge\left(-\mathrm{d} \beta \wedge *\left(\mathrm{~d} \beta \wedge \theta_{a}\right)-*\left(* \mathrm{~d} \beta \wedge \theta_{a}\right) \wedge * \mathrm{~d} \beta\right), \\
\delta( & \beta \mathrm{d} \beta \wedge * Q)=-\delta \beta \cdot \beta \mathrm{d} * Q+\mathrm{d}\left(\beta \delta \beta * Q-\delta g_{a b} \beta g^{a b} * \mathrm{~d} \beta\right)+\delta \Gamma^{a}{ }_{b} \wedge 2 \beta \delta_{a}^{b} * \mathrm{~d} \beta \\
+ & \delta g_{a b}\left(\beta\left(g^{a b} \mathrm{D} * \mathrm{~d} \beta+\frac{1}{2} g^{a b} \mathrm{~d} \beta \wedge * Q-\theta^{(a} *\left(* Q \wedge \theta^{b)}\right) * \mathrm{~d} \beta\right)\right. \\
+ & \delta \theta^{a} \wedge \beta\left(-\mathrm{d} \beta \wedge *\left(Q \wedge \theta_{a}\right)-*\left(* Q \wedge \theta_{a}\right) * \mathrm{~d} \beta\right),
\end{aligned}
$$

As a result of the variational procedure using the Lemma, we have obtained three field equations: $\Gamma$-equation, $\theta$-equation, and $\beta$-equation:

$\Gamma$-equation:

$$
\begin{aligned}
& f_{0}\left[-(1 / 4) Q \wedge \eta_{a}{ }^{b}+(1 / 2) \mathcal{T}_{c} \wedge \eta_{a}{ }^{b c}+(1 / 2) \eta_{a c} \wedge Q^{b c}+\mathrm{d} \ln \beta \wedge \eta_{a}{ }^{b}\right. \\
& +2 \rho_{1} \theta^{b} \wedge * \mathcal{T}_{a}+2 \rho_{2} \theta^{b} \wedge \theta_{c} \wedge *\left(\mathcal{T}^{c} \wedge \theta_{a}\right)+2 \rho_{3} \theta^{b} \wedge \theta_{a} \wedge *\left(\mathcal{T}^{c} \wedge \theta_{c}\right) \\
& +4 \xi \delta_{a}^{b}[2,3,4] Q+\zeta\left(2 \delta_{a}^{b} \theta^{c} \wedge * \mathcal{T}_{c}+\theta^{b} \wedge *\left(Q \wedge \theta_{a}\right)\right) \\
& \left.+l_{2} \theta^{b} \wedge *\left(\mathrm{~d} \ln \beta \wedge \theta_{a}\right)+l_{3} 2 \delta_{a}^{b} * \mathrm{~d} \ln \beta\right]-\beta^{2} \Lambda_{a}{ }^{b}=0 .
\end{aligned}
$$

$\theta$-equation:

$$
\begin{aligned}
& \frac{1}{2} \mathcal{R}^{b}{ }_{c} \wedge \eta_{b}{ }^{c} a-\beta^{2} \Lambda_{0} \eta_{a} \\
& +\rho_{1}\left[2 \mathrm{D} * \mathcal{T}_{a}+\mathcal{T}_{b} \wedge *\left(\mathcal{T}^{b} \wedge \theta_{a}\right)+*\left(* \mathcal{T}^{b} \wedge \theta_{a}\right) \wedge * \mathcal{T}_{b}+4 \mathrm{~d} \ln \beta \wedge * \mathcal{T}_{a}\right] \\
& +\rho_{2}\left[2 \mathrm{D}\left(\theta_{b} \wedge *\left(\mathcal{T}^{b} \wedge \theta_{a}\right)\right)+2 \mathcal{T}^{b} \wedge *\left(\theta_{b} \wedge \mathcal{T}_{a}\right)-*\left(\mathcal{T}^{b} \wedge \theta_{c} \wedge \theta_{a}\right)\left(\mathcal{T}^{c} \wedge \theta_{b}\right)\right. \\
& \left.-*\left(*\left(\mathcal{T}^{c} \wedge \theta_{d}\right) \wedge \theta_{a}\right) \wedge *\left(\mathcal{T}^{d} \wedge \theta_{c}\right)+4 \mathrm{~d} \ln \beta \wedge \theta_{b} \wedge *\left(\mathcal{T}^{b} \wedge \theta_{a}\right)\right] \\
& +\rho_{3}\left[2 \mathrm{D}\left(\theta_{a} \wedge *\left(\mathcal{T}^{b} \wedge \theta_{b}\right)\right)+2 \mathcal{T}_{a} \wedge *\left(\mathcal{T}^{b} \wedge \theta_{b}\right)-*\left(\mathcal{T}^{b} \wedge \theta_{b} \wedge \theta_{a}\right)\left(\mathcal{T}^{c} \wedge \theta_{c}\right)\right. \\
& \left.-*\left(*\left(\mathcal{T}^{b} \wedge \theta_{b}\right) \wedge \theta_{a}\right) \wedge *\left(\mathcal{T}^{c} \wedge \theta_{c}\right)+4 \mathrm{~d} \ln \beta \wedge \theta_{a} \wedge *\left(\mathcal{T}^{b} \wedge \theta_{b}\right)\right] \\
& +\xi\left[-Q \wedge *\left(Q \wedge \theta_{a}\right)-*\left(* Q \wedge \theta_{a}\right) * Q\right]+\zeta\left[\mathrm{D} *\left(Q \wedge \theta_{a}\right)-Q \wedge * \mathcal{T}_{a}+Q \wedge \theta^{b} \wedge *\left(\mathcal{T}_{b} \wedge \theta_{a}\right)\right. \\
& \left.+*\left(* \mathcal{T}_{b} \wedge \theta_{a}\right) \wedge *\left(Q \wedge \theta^{b}\right)+2 \mathrm{~d} \ln \beta \wedge *\left(Q \wedge \theta_{a}\right)\right] \\
& \left.+l_{1}\left[-\mathrm{d} \ln \beta \wedge *\left(\mathrm{~d} \ln \beta \wedge \theta_{a}\right)-*\left(* \mathrm{~d} \ln \beta \wedge \theta_{a}\right) \wedge * \mathrm{~d} \ln \beta\right)\right] \\
& +l_{2}\left[\mathrm{D} *\left(\mathrm{~d} \ln \beta \wedge \theta_{a}\right)+\mathrm{d} \ln \beta \wedge \theta^{b} \wedge *\left(\mathcal{T}_{b} \wedge \theta_{a}\right)-\mathrm{d} \ln \beta \wedge * \mathcal{T}_{a}+* * \mathcal{T}_{b} \wedge \theta_{a}\right) \wedge *\left(\mathrm{~d} \ln \beta \wedge \theta^{b}\right) \\
& \left.+2 \mathrm{~d} \ln \beta \wedge *\left(\mathrm{~d} \ln \beta \wedge \theta_{a}\right)\right]+l_{3}\left[-\mathrm{d} \ln \beta \wedge *\left(Q \wedge \theta_{a}\right)-* *\left(* \theta_{a}\right) * \mathrm{~d} \ln \beta\right]=0 .
\end{aligned}
$$


$\beta$-equation:

$$
\begin{aligned}
& \mathcal{R}^{a}{ }_{b} \wedge \eta_{a}{ }^{b}-4 \beta^{2} \Lambda_{0} \eta+\rho_{1} 2 \mathcal{T}^{a} \wedge * \mathcal{T}_{a}+\rho_{2}\left(2 \mathcal{T}^{a} \wedge \theta_{b}\right) \wedge *\left(\mathcal{T}^{b} \wedge \theta_{a}\right) \\
& +\rho_{3} 2\left(\mathcal{T}^{a} \wedge \theta_{a}\right) \wedge *\left(\mathcal{T}^{b} \wedge \theta_{b}\right)+\xi 2 Q \wedge * Q+\zeta 2 Q \wedge \theta^{a} \wedge * \mathcal{T}_{a} \\
& +l_{1}(-2 \mathrm{~d} * \mathrm{~d} \ln \beta-2 \mathrm{~d} \ln \beta \wedge \mathrm{d} \ln \beta)+l_{2}\left(-\mathrm{d}\left(\theta^{a} \wedge * \mathcal{T}_{a}\right)\right)+l_{3}(-\mathrm{d} * Q)=0 .
\end{aligned}
$$

Varying the Lagrangian density (1) with respect to the indefinite Lagrange multipliers $\Lambda^{a b}$ leads to the Weyl condition for nonmetricity:

$$
\boldsymbol{Q}_{a b}-(1 / 4) g_{a b} \mathbf{Q}=0 .
$$

It turns out that the variational equation corresponding to the variation in the components of the metric tensor $g_{a b}$ is a consequence of the remaining variational Equations (8)-(11). This fact is a consequence of the used variational principle.

If we compare the obtained variational field Equations (8)-(10) with similar equations arising, for example, in the well-known Einstein-Cartan theory of gravity, then Equation (8) will be an analogue of the equation, the source in which in the Einstein-Cartan theory will be the spin moment tensor of external fields and media. However, in our case, Equation (8) has a broader meaning and also reflects the dependence on the dilatation current.

The variational field Equation (9) is a generalization of Einstein's equation in tetrad form. The source in this equation is the canonical energy-momentum tensor of external fields and media. In our case, these additional sources are absent. The variational field Equation (10) is a new equation arising only in the Poincaré-Weyl gauge theory of gravitation.

We obtained some results from the $\Gamma$-equation. For this, we antisymmetrize the $\Gamma$-equation (8), dropping the subscript $b$ down and changing the sign. We obtain an equation that does not contain indefinite Lagrange multipliers:

$$
\begin{aligned}
& \frac{1}{8} Q \wedge \eta_{a b}-\frac{1}{2} \mathcal{T}^{c} \wedge \eta_{a b c}-\operatorname{dln} \beta \wedge \eta_{a b}+2 \rho_{1} \theta_{[a} \wedge * \mathcal{T}_{b]}+2 \rho_{2} \theta_{[a} \wedge \theta_{|c|} \wedge *\left(\mathcal{T}^{c} \wedge \theta_{b]}\right) \\
& +2 \rho_{3} \theta_{a} \wedge \theta_{b} \wedge *\left(\mathcal{T}^{c} \wedge \theta_{c}\right)+\zeta \theta_{[a} \wedge *\left(Q \wedge \theta_{b]}\right)+l_{2} \theta_{[a} \wedge *\left(\operatorname{dln} \beta \wedge \theta_{b]}\right)=0 .
\end{aligned}
$$

Multiplying this equation externally by $\theta^{b}$ on the right and considering the expressions for the 1-form of the torsion trace and its dual conjugation:

$$
\mathcal{T}=*\left(\theta_{b} \wedge * \mathcal{T}^{b}\right), * \mathcal{T}=* *\left(\theta_{b} \wedge * \mathcal{T}^{b}\right)=(-1)^{3(4-3)+3} \theta_{b} \wedge * \mathcal{T}^{b}=\theta_{b} \wedge * \mathcal{T}^{b},
$$

We obtain a simpler version of the $\Gamma$-equation, which does not contain the indefinite Lagrange multipliers:

$$
2\left(\rho_{1}-2 \rho_{2}-1\right) \mathcal{T}+3(1 / 4+\zeta) Q=\left(6-3 l_{2}\right)(\mathrm{d} \beta / \beta)
$$

Another corollary of the $\Gamma$-equation, which does not contain indefinite Lagrange multipliers, can be obtained by convolving the $\Gamma$-equation by indices $a, b$, taking into account that $\Lambda_{a}^{a}=0$. As a result of the convolution, we obtain:

$$
2\left(\rho_{1}-2 \rho_{2}+4 \zeta\right) \mathcal{T}+(16 \xi+3 \zeta) Q=\left(-3 l_{2}-8 l_{3}\right)(\mathrm{d} \beta / \beta) .
$$




\section{Cosmological Solution for the Primordial Universe}

Let us consider the homogeneous, isotropic, and spatially flat Universe with the Friedman-Robertson-Walker (FRW) metrics:

$$
d s^{2}=d t^{2}-a^{2}(t)\left(d x^{2}+d y^{2}+d z^{2}\right)
$$

and let us solve for this metrics the Poincaré-Weyl gravity field Equations (8)-(10) for the scale invariant primordial stage of the Universe evolution.

It is known from previous works [20-22,29-31] that for this metric, the 2-form of torsion has the form, $\mathcal{T}_{a}=(1 / 3) \mathcal{T} \wedge \theta_{a}$, and is completely determined by the 1-form of its trace $\mathcal{T}=*\left(\theta_{c} \wedge * \mathcal{T}^{a}\right)$. As a consequence of Equations (14) and (15), we can conclude that the 1-form of the torsion trace and the 1-form of the trace of nonmetricity are determined only by the differential of the natural logarithm of the scalar Weyl-Dirac field:

$$
\mathcal{T}=s(\mathrm{~d} \beta / \beta), Q=q(\mathrm{~d} \beta / \beta)
$$

here, the numbers $s, q$ are determined by the coupling constants of the Lagrangian density (1). In what follows, we use the condition:

$$
\frac{q}{8}-\frac{s}{3}=1
$$

which has been also accepted when finding spherically and axially symmetric solutions of the Poincaré-Weyl theory of gravity [29-31].

As a result, we obtain that the only non-null expressions of the $\theta$-equation (9) are:

$$
\begin{gathered}
3 H^{2}+6 H U+U^{2}\left(k^{2}+3\right)=\Lambda_{0} \beta^{2}, \\
2 H_{t}+2 U_{t}+4 H U+3 H^{2}-U^{2}\left(k^{2}-1\right)=\Lambda_{0} \beta^{2},
\end{gathered}
$$

where we have introduced notations:

$$
\begin{gathered}
k^{2}=l_{1}+(1 / 2) s l_{2}+(1 / 2) q l_{3}, \\
H=a_{t} / a, \quad a_{t}=d a / d t, \quad U=\beta_{t} / \beta, \quad \beta_{t}=d \beta / d t,
\end{gathered}
$$

and the only non-null expression of the $\beta$-equation (10) is:

$$
3 H_{t}+6 H^{2}+\left(U_{t}+U^{2}+3 H U\right)\left(k^{2}+3\right)=2 \Lambda_{0} \beta^{2} .
$$

Now, we solve the system of equations obtained. Let us subtract Equation (19) from Equation (20). As a result, we obtain:

$$
H_{t}+U_{t}-H U-U^{2}\left(k^{2}+1\right)=0 .
$$

Then, after subtracting double Equation (20) from Equation (23) and taking into account Equation (24), we obtain:

$$
k^{2}\left(U_{t}+3 H U+2 U^{2}\right)=0, \quad k^{2} \neq 0 .
$$

when $k^{2} \neq 0$, we obtain for two unknown functions $a(t)$ and $\beta(t)$ three equations: (19), (24), and (25). Thus, the system of equations is over-determined.

Let us estimate the value of $k^{2}$. Based on the calculation of the flyby anomaly (upon receipt of the spherically symmetric solution $[29,30])$, the following estimate was obtained:

$$
k^{2} \approx 10^{-20} \div 10^{-22} \text {. }
$$

Therefore, in Equations (19) and (24), we may neglect the terms with the coefficient $k^{2}$. Then, Equation (19) will be reduced to the equation: 


$$
H+U=(\lambda / 3) \beta, \quad \lambda=\sqrt{3 \Lambda_{0}} .
$$

Here, from the two possible signs, we take the " + " sign.

In this case, Equation (24), subject to Equation (26), turns out to be a consequence of equation (27). The system of equations reduces to the two Equations (25) and (27) for two unknown functions $a(t)$ and $\beta(t)$, and the system of equations becomes well defined.

It is easy to understand that in case $k^{2}=0$, the system of Equations (19), (24), and (25) turns out to be underdetermined, because in this case, there is only one Equation (27) for two unknown functions.

Equation (25), subject to Equation (27), is reduced to the equation:

$$
U_{t}+\lambda \beta U-U^{2}=0
$$

which is equivalent to the following equation:

$$
\beta \beta_{t t}+\lambda \beta_{t} \beta^{2}-\beta_{t}^{2}=0 .
$$

which is integrated by the substitution $\beta_{t}=\beta^{2} z(\beta)$. The first integral of this equation is:

$$
U=\beta_{t} / \beta=-\lambda \beta \ln (C \beta) .
$$

here, $C$ is the constant of integration.

We obtain the second equation of the system after substituting this solution (30) into Equation (27):

$$
H=a_{t} / a=\lambda \beta(\ln (C \beta)+1 / 3) .
$$

A boundary condition is:

$$
C \beta \rightarrow 1 \text { when } t \rightarrow \infty .
$$

The approach to the limit value $C \beta \rightarrow 1$ for large $t$ occurs exponentially, $C \beta-1 \sim e^{\frac{-\lambda t}{C}}$.

Then, we impose a requirement that the value of the effective cosmological constant $\beta^{2}(t) \Lambda_{0}$ coincides with the modern value of the cosmological constant:

$$
\beta^{2}\left(t_{U}\right) \Lambda_{0}=\Lambda, t_{U}=13.8 \text { billion years }
$$

where $t_{U}$ is the Universe lifetime. This requirement determines the value of the constant $S$.

The result of the numerical integration of the system of Equations (30) and (31) with the boundary condition (32) is presented graphically in Figures 1 and 2, where it can be seen for small and large times $t$, the inflationary behavior of the scale factor $a(t)$, as well as for small times $t$, there is a sharp exponential decrease of the Weyl-Dirac scalar field $\beta$; therefore, the effective cosmological constant $\beta^{2}(t) \Lambda_{0}$ decreases from a huge value at the beginning of the Big Bang to an extremely small (but not zero) value in the modern epoch, which coincides with its observed value.

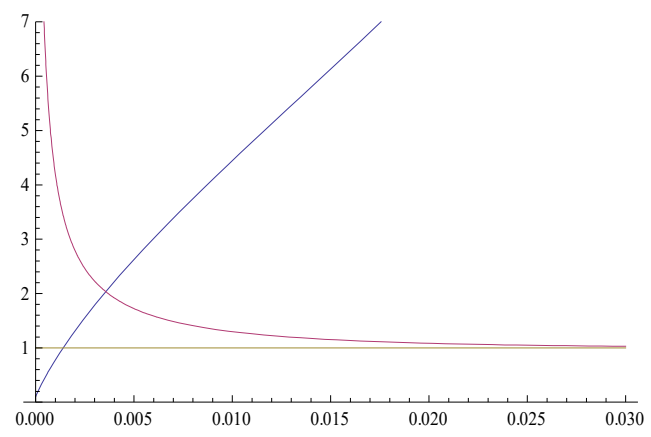

Figure 1. Model of behavior of the functions $a(t)$ and $S \beta(t)$ at small values of time $t$. 


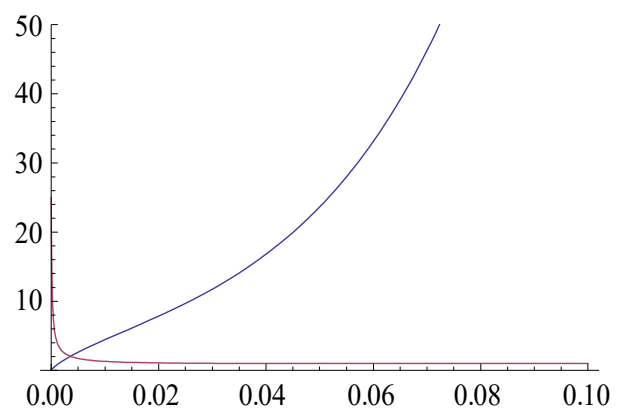

Figure 2. Model of behavior of the functions $a(t)$ and $S \beta(t)$ at large values of time $t$.

\section{Conclusions}

One can conclude following the idea by Harrison and Zel'dovich that the Universe at the early stage has the property of scale invariance [23]. In this paper, we have proposed that the symmetry group of the space-time at the primordial stage of the Universe is the Poincaré-Weyl group, and space-time is the Cartan-Weyl space with a curvature and torsion of 2-forms, as well as a nonmetricity 1-form with the Weyl condition. Furthermore, in addition to the metric tensor, a Weyl-Dirac scalar field $\beta$ appears, which determines the dark energy amount.

According to Gliner, the Lagrangian density of the theory contains a vacuum-like term, which in the proposed theory depends on the Weyl-Dirac scalar field and is interpreted as a term with an effective cosmological constant (dark energy term).

The theory constructed is applicable to the primordial stage of a homogeneous Universe with the FRW metrics with a scale factor $a(t)$. We obtain two Equations, (30) and (31), and using the numerical integration, we obtain the sharp exponential decrease of the effective cosmological constant $\beta^{2} \Lambda_{0}$ at small $t$ and the exponential increase of the scale factor [32]. In the proposed theory, the inflation problem acquires new features: the Weyl-Dirac scalar field becomes the inflation and inflation becomes scale-invariant. For the meaning of the latter circumstance, see [23].

The result obtained can be considered as a solution to one of the main contradictions of the theory of the Universe evolution, which is called the "problem of the cosmological constant" [27,33-38]. The essence of this problem is a huge (about 120 order) difference between the calculated value of the cosmological constant in the initial stage of the Universe evolution and its value, determined on the basis of modern observational data.

The hypothesis on the sharply decrease of the effective cosmological constant as a consequence of the fields dynamics in the primordial Universe was expressed in [19-22]. The main thing of the solutions obtained in [19-22,32], and also in this paper, is that these solutions demonstrate a sharp decrease in the effective cosmological constant at a small $t$ and approach the modern value of the cosmological constant (but not zero) at large values of $t$.

Author Contributions: The contributions of both authors to the results of the article are substantial and equivalent. All authors have read and agreed to the published version of the manuscript.

Funding: This research received no external funding.

Conflicts of Interest: The authors declare no conflict of interest.

\section{References}

1. Kibble, T.W.B. Lorentz invariance and the gravitational field. J. Math. Phys. 1961, 2, 212-221. [CrossRef]

2. Trautman, A. Recent advances in the Einstein-Cartan theory of gravity. Ann. N. Y. Acad. Sci. 1975, 262, 241-245. [CrossRef]

3. Frolov, B.N. Principle of local invariance and Noether theorem. Vestn. Mosk. Univ. Ser. Fiz. Astron. 1963, 6, 48-58. (In Russian) 
4. Frolov, B.N. Principle of local invariance and Noether theorem. In Modern Problems of Gravitation/Proceedings of the 2nd Soviet Gravitation Conference; Publishing House of Tbilisi University: Tbilisi, Georgia, 1967; pp. 270-278. (In Russian)

5. Frolov, B.N.; Sardanashvily, G.A. Gravitation and gauge fields. Sov. J. Phys. 1974, 17, 1228-1231. [CrossRef]

6. Hayashi, K. Extended translational invariance and associated gauge fields. Progr. Theor. Phys. 1967, 38, 491-507. [CrossRef]

7. Hayashi, K. Gauge theories of massive and massless tensor fields. Progr. Theor. Phys. 1968, 39, $495-515$. [CrossRef]

8. Hehl, F.W.; von der Heyde, P.; Kerlick, G.D.; Nester, J.M. General relativity with spin and torsion: Foundtion and prospects. Revs. Mod. Phys. 1976, 48, 393-416. [CrossRef]

9. Blagojevic, M. Gravitation and Gauge Symmetries; Institute Physics Publishing IOP Publishing Ltd.: Bristol, UK; Philadelphia, PA, USA, 2002.

10. Frolov, B.N. Poincar'e-Gauge Theory of Gravity; Prometej Publishing House: Moscow, Russia, 2003. (In Russian)

11. Frolov, B.N. On foundations of Poincar'e-gauge theory of gravity. Grav. Cosmol. 2004, 10, 116-120.

12. Ponomarev, V.N.; Barvinsky, A.O.; Obukhov, Y.N. Gauge Approach and Quantization Methods in Gravity Theory; Nauka: Moscow, Russia, 2017; p. 360.

13. Charap, J.M.; Tait, W. A gauge theory of the Weyl group. Proc. R. Soc. 1974, A340, $249-262$.

14. Kasuya, M. Gauge theory in the Einstein-Cartan-Weyl space-time. Nuovo Cim. 1975, 28B, 127-137. [CrossRef]

15. Babourova, O.V.; Frolov, B.N.; Zhukovsky, V.C. Gauge Field Theory for Poincare-Weyl Group. Phys. Rev. 2006, 74, 064012. [CrossRef]

16. Babourova, O.V.; Zhukovskii, V.C.; Frolov, B.N. A Weyl-Cartan space-time model based on the gauge principle. Theor. Math. Phys. 2008, 157, 1420-1432. [CrossRef]

17. Babourova, O.V.; Frolov, B.N.; Zhukovsky, V.C. Theory of Gravitation on the Basis of the Poincare-Weyl Gauge Group. Gravit. Cosmol. 2009, 15, 13-15. [CrossRef]

18. Dirac, P.A.M. Long range forces and broken symmetries. Proc. R. Soc. 1973, A333, 403-418.

19. Babourova, O.V.; Frolov, B.N. Dark energy, Dirac's scalar field and the cosmological constant problem. arXiv 2011, arXiv:1112.4449.

20. Babourova, O.V.; Frolov, B.N. Mathematical Problems of the Modern Theory of Gravity; MPSU Press: Moscow, Russia, 2012. (In Russian)

21. Babourova, O.V.; Frolov, B.N.; Lipkin, K.N. Theory of gravitation with scalar Dirac field in exterior form formalism and the cosmological constant problem. Gravit. Cosmol. 2012, 18, 225-231. [CrossRef]

22. Babourova, O.V.; Frolov, B.N. Dark Energy as a Cosmological Consequence of Existence of the Dirac Scalar Field in Nature. Phys. Res. Intern. 2015, 2015, 952181. [CrossRef]

23. Rubakov, V.A. Harrison-Zel'dovich spectrum from conformal invariance. arXiv 2009, arXiv:0906.3693.

24. Sazhin, M.V. Anisotropy and polarization of cosmic microwave background: State of the art. Phys. Uspekhi. 2004, 47, 187-194. [CrossRef]

25. Gliner, E.B. Algebraic properties of the energy-momentum tensor and vacuum-like states of matter. Sov. Phys. J. Exp. Theor. Phys. 1966, 22, 378-382.

26. Gliner, E.B. Inflationary universe and the vacuumlike state of physical medium. Phys. Uspekhi. 2002, 45, 213-220. [CrossRef]

27. Weinberg, S. The cosmological constant problem. Rev. Mod. Phys. 1989, 61, 1-23. [CrossRef]

28. Babourova, O.V.; Frolov, B.N.; Klimova, E.A. Plane torsion waves in quadratic gravitational theories in Riemann-Cartan space. Class. Quantum Grav. 1999, 16, 1149-1162. [CrossRef]

29. Babourova, O.V.; Frolov, B.N.; Febres, E.V. Spherically symmetric solution of gravitation theory with a Dirac scalar field in the Cartan-Weyl space. Russ. Phys. J. 2015, 57, 1297-1299. [CrossRef]

30. Babourova, O.V.; Frolov, B.N.; Kudlaev, P.E.; Romanova, E.V. Spherically symmetric solution of the Weyl-Dirac theory of gravitation and possible influence of dark matter on the interplanetary spacecraft motion. arXiv 2016, arXiv:1610.09525.

31. Babourova, O.V.; Frolov, B.N.; Kudlaev, P.E. Axially Symmetric Solution of the Weyl-Dirac Theory of Gravitation and the Problem of Rotation Curves of Galaxies. Gravit. Cosmol. 2018, 24, 118-121. [CrossRef]

32. Babourova, O.V.; Frolov, B.N. Harrison-Zel'dovich scale invariance and the exponential decrease of the "cosmological constant" in the super-early Universe. arXiv 2020, arXiv:2001.05968.

33. Weinberg, S. The Cosmological Constant Problems. arXiv 2000, arXiv:0005265v1. 
34. Peebles, P.J.E.; Ratra, B. The cosmological constant and dark energy. Rev. Mod. Phys. 2003, 75, 559-606. [CrossRef]

35. Li, M.; Li, X.-D.; Wang, S.; Wang, Y. Dark Energy. Commun. Theor. Phys. 2011, 56, 525-604. [CrossRef]

36. Gorbunov, D.S.; Rubakov, V.A. Introduction to the Theory of the Early Universe: Hot Big Bang Theory; World Scientific: Singapore, 2011.

37. Martin, J. Everything you always wanted to know about the cosmological constant problem (but were afraid to ask). C. R. Phys. 2012, 13, 566-665. [CrossRef]

38. Luongo, O.; Muccino, M. Speeding up the universe using dust with pressure. Phys. Rev. 2018, 98, 103520. [CrossRef]

Publisher's Note: MDPI stays neutral with regard to jurisdictional claims in published maps and institutional affiliations.

(C) 2020 by the authors. Licensee MDPI, Basel, Switzerland. This article is an open access article distributed under the terms and conditions of the Creative Commons Attribution (CC BY) license (http://creativecommons.org/licenses/by/4.0/). 